\title{
A Nonlinear Contour Preserving Transform for Geometrical Image Compression
}

\author{
W. Van Aerschot \\ Computer Science Dept., K.U.Leuven \\ Celestijnenlaan $200 \mathrm{~A}$, \\ B3001 Heverlee - Belgium, \\ Ward.VanAerschot@cs.kuleuven.be
}

\author{
M. Jansen \\ Department of Math., K.U.Leuven \\ Celestijnenlaan $200 \mathrm{~B}$, \\ B3001 Heverlee - Belgium, \\ Maarten.Jansen@wis.kuleuven.be
}

\author{
A. Bultheel \\ Computer Science Dept., K.U.Leuven \\ Celestijnenlaan $200 \mathrm{~A}$, \\ B3001 Heverlee - Belgium, \\ Adhemar.Bultheel@cs.kuleuven.be
}

\begin{abstract}
Recently the performance of nonlinear transforms have been given a lot of attention to overcome the suboptimal nterms approximation power of tensor product wavelet methods on higher dimensions. The suboptimal performance prevails when those transforms are used for a sparse representation of functions consisting of smoothly varying areas separated by smooth contours. This paper introduces a method creating normal meshes with nonsubdivision connectivity to approximate the nonsmoothness of such images efficiently. From a domain decomposition viewpoint, the method is a triangulation refinement method preserving contours. The so-called normal offset decomposition searches from the midpoint of the edges in the previous approximation along the normal direction until it pierces the surface that represents the image and adds the piercing points to the approximation. The transform is nonlinear as it depends on the actual image. In this paper, we propose a normal offset based compression algorithm for digital images. The discrete setting causes the transform to become redundant. We also propose a model to encode the obtained coefficients. We show rate distortion curves and compare the results with the JPEG2000 encoder.
\end{abstract}

\section{Introduction}

Normal meshes have been introduced as a method for surface representation in computer graphics applications. They allow sparse representations of surfaces approximating enormous amounts of data coming from scanning smooth objects $[2,8,10,12]$. Normal meshes have been successfully introduced in this field. Each vertex can be represented by one coefficient in a local frame instead of its usual three coordinates. This property makes normal meshes also suited for mesh compression [12]. A more theoretical study investigates the approximation of smooth curves with polylines [4].

This paper investigates the application of a nonlinear transform based on normal mesh techniques to compress images whose content is dominated by contours. These kind of images will be referred to as geometrical images (see Figure 1). For this type of images, transform coders using nonredundant bases fall short when creating compact high resolution representations. The highest achievable $n$-term approximation rate using a wavelet transform coder combined with a nonlinear thresholding equals $\sigma_{L_{2}}=O\left(n^{-\frac{1}{2}}\right)$ [6]. Therefore, transform coders like JPEG2000 will perform suboptimally compared to recently developed schemes capable of sparsely representing line discontinuities (see Figure 2 for an example).

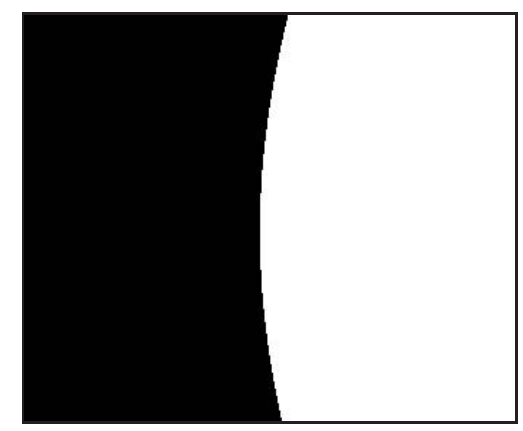

Figure 1. Geometrical Image.

These schemes can be classified into two categories. On the one hand, redundant linear transforms, like directional frames, combined with a nonlinear $n$-term selection e.g. curvelets [3], contourlets [7]. On the other hand, we have highly nonlinear schemes where also the transform is nonlinear. Examples are geometric wavelets [5], bandelets [11], dictionary approaches and so on. Also the normal offset scheme belongs to this category. The nonlinearity of the transform partitions the image domain into parts of different sizes and shapes. The small parts correspond to image regions that contain much information and the large parts to 


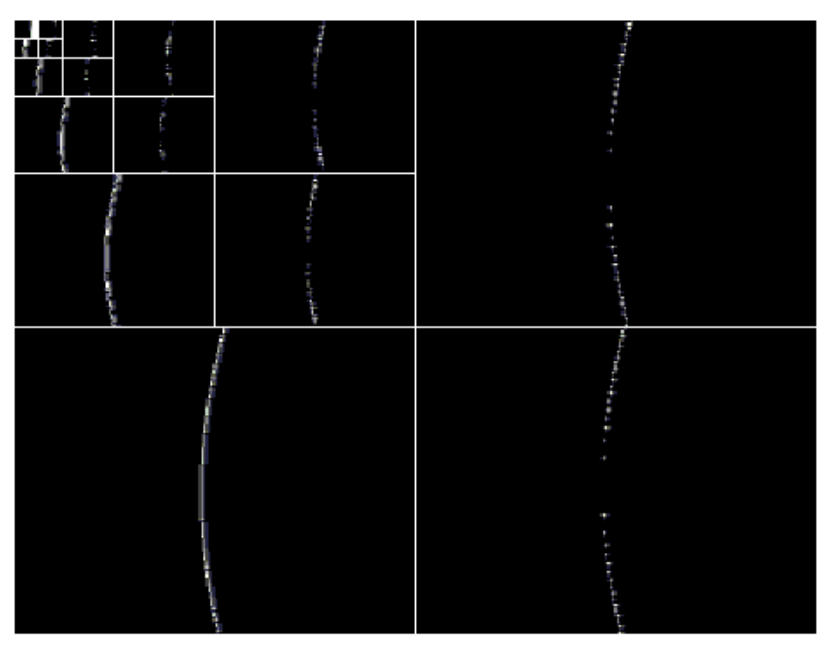

Figure 2. Two dimensional wavelet decomposition at level 5 . The grayscale is dependent on the magnitude of the waveletcoefficients. The contour is cleary visible in all subbands in each direction (horizontal, vertical and diagonal subbands). This illustrates the large amount of coefficient needed to catch line singularities which results in the nonsparseness of a wavelet representation of geometrical images.

smooth image regions. The goal is to let the contour be approximated by the borders of the parts situated in the neighborhood.

The paper is outlined as follows. Section 1.1 explains the difference with normal meshes used to represent smooth $3 \mathrm{D}$ surfaces and image compression. Section 2 comments on the used multiresolution structure. In Section 3 the proposed normal mesh algorithm is given in detail. This nonlinear transform forms the main component of the encoder. It produces sparse representations of geometrical images. The efficient encoding of the wavelet coefficients (normal offsets) is done by a model based entropy coder and will be explained in Section 5. Section 4 compares the approximation rate of the proposed transform and the wavelet transform. Finally Section 6 shows rate-distortion curves of the proposed encoder and compares the results with the state of the art JPEG2000 encoder.

\subsection{Smooth versus nonsmooth manifolds}

An analogue grayscale image $\mathcal{I}$ is defined as a real function on the square, i.e. $\mathcal{I}(x, y):[0,1]^{2} \mapsto R^{2}$. Images represented as functions on a 2 -d plane can also be seen as surfaces in $R^{3}$. Despite the similarities between surface and image compression we cannot take normal mesh encoders and apply them to the surface equivalents of a geometrical image. The first major difference concerns the smoothness properties of the target surfaces. While surfaces in computer graphics are smooth, geometrical images on the other hand are non-smooth: the line discontinuities are the information carrying feature. A second major difference - and the actual reason for using a normal offset transform in an image compression algorithm-is the exploitation of normal approximation properties in order to compress. The reason for using normal offsets in surface rendering applications is that they contain almost all geometrical information [8]. Grace to the minor contribution of the parametrical information, semi-regular meshes, demanding almost no connectivity information can be utilized. As such, the surface can be approximated by a mesh where each point is defined using a single scalar. This leads to a bonus compression factor of three. Image grey levels are not triple coordinates in $R^{3}$, but single values on a given, regular lattice, so there is no bonus to gain in the image case. Irregular meshes have to be used to preserve contours. The fundamental reason for using normal offsets in image processing is the edge locating property of the normal search direction. The application of normal offsets to images leads to a different implementation.

A first attempt to use normal mesh techniques for image approximation was made in [9]. Gray scale images are treated as two dimensional functions dominated by geometric structures comparable with terrain models used in geographical information systems. The authors achieve an $n$ term approximation rate that is twice as good as wavelet approximations on the studied images. They do not address compression, but since approximation and compression are tightly related, their results indicate that the normal offset method should be considered for the development of ratedistortion efficient image encoders.

\section{Multiresolution model}

The multi-resolution model used in [9] is non nested. Therefore additional information needs to be stored in order to encode the complex dependency relations between consecutive resolution levels. They do not incorporate a contour preserving triangulation method which explains the poor visual results of the presented transform.

We propose a nested multi-resolution model with a more adequate definition of the normal direction w.r.t. compression of non-smooth functions instead of smooth surfaces. We use local mesh refinements with additional adaptivity to preserve the approximation strength. The use of local mesh refinements results in tree-structured dependency relations between successive approximations. The refinements are 
simple triangle splits that can be encoded in a straightforward manner.

\section{The algorithm}

We now give a specific implementation of each step the normal offset transformation scheme:

\subsection{Prediction step}

Let $\mathcal{E}_{j}$ be the set of edges of the coarse mesh. The for each $e_{j, k} \in \mathcal{E}_{j}$ a prediction point is calculated as

$$
p_{j+1,2 k+1}^{*}:=\frac{p_{j, k}+p_{j, k+1}}{2},
$$

with $p_{j, k}$ and $p_{j, k+1}$ the endpoints of $e_{j, k}$.

\subsection{Correction step}

The correction step corrects the location of the prediction points by adding a vector in the direction normal to the coarse mesh. The normals $\vec{n}_{j, k}$, on the coarse mesh such that $\left\|\vec{n}_{j, k}\right\|=1$ are expressed as

$\vec{n}_{j, k}=\left[\begin{array}{c}n_{[x]_{j, k}} \\ n_{[y]_{j, k}}\end{array}\right]:=\frac{\left[-\left(y_{j, k+1}-y_{j, k}\right) x_{j, k+1}-x_{j, k}\right]^{T}}{\left\|p_{j, k+1}-p_{j, k}\right\|}$,

and represents the direction of the perpendicular bisector on $e_{j, k}$. The normal ray $r_{j+1,2 k+1}$ going through $p_{j+1,2 k+1}^{*}$ is defined as:

$$
r_{j+1,2 k+1}(\gamma):=p_{j+1,2 k+1}^{*}+\gamma \vec{n}_{j, k}
$$

For $f \in \mathcal{C}$, with $\mathcal{C}$ the class of continuous functions, the correction step calculates

$$
\gamma_{j+1,2 k+1}:=\min \left\{\gamma \mid r_{j+1,2 k+1}(\gamma)=\left[x, f_{e}(x)\right]\right\} .
$$

The minimum value of $\gamma$ over a set is taken, since in general the normal ray can pierce $f_{e}$ more than once.

For $f \notin \mathcal{C}$ and $r$ parameterized as $r_{j+1,2 k+1}(\gamma)=$ $\left(x_{j+1,2 k+1}(\gamma), y_{j+1,2 k+1}(\gamma)\right)$ Equation 3 takes on following form:

$$
\begin{array}{r}
\gamma_{j+1,2 k+1}:=\min \{\gamma \mid \\
\operatorname{sign}\left(\lim _{x_{j+1,2 k+1}(\gamma) \rightarrow x^{+}} y_{j+1,2 k+1}(\gamma)-f_{e}(x)\right) \\
\left.=-\operatorname{sign}\left(\lim _{x_{j+1,2 k+1}(\gamma) \rightarrow x^{-}} y_{j+1,2 k+1}(\gamma)-f_{e}(x)\right)\right\}
\end{array}
$$

As such we always obtain a subdivision scheme i.e. : $x_{j, k} \leq x_{j+1,2 k+1} \leq x_{j, k+1}$. Setting $x_{j+1,2 k}:=x_{j, k}$ we obtain a monotonically increasing sequence $\mathbf{x}_{j}=$ $\left\{x_{j, k}, x_{j, k+1}, x_{j, k+2}, \cdots\right\}$.

The point $p_{j+1,2 k+1}:=r_{j+1,2 k+1}\left(\gamma_{j+1,2 k+1}\right)$ is called a piercing point. The piercing point $p_{j+1,2 k+1}$ is inserted between $p_{j, k}$ and $p_{j, k+1}$ in order to form the sequence $\mathbf{p}_{j+1}$ :

$$
\begin{array}{rc}
p_{j+1,2 k} & \leftarrow p_{j, k} \\
p_{j+1,2 k+1} & \leftarrow r_{j+1,2 k+1}\left(\gamma_{j+1,2 k+1}\right) . \\
p_{j+1,2 k+2} & \leftarrow p_{j, k+1}
\end{array}
$$

The function above $e_{j, k}$ is gradually approximated by a polyline (a continuous curve composed of several line segments) through the sequence $\mathbf{p}_{j}=$ $\left\{p_{j, 0}, \ldots, p_{j, k} \ldots, p_{j, 2^{j}+1}\right\}$.

The physical interpretation of $\gamma_{j, k}$ is the $y^{\prime}$-coordinate of the piercing point $p_{j, k}$ located in the local coordinate system $x^{\prime} y^{\prime}$ with origin $p_{j, k}^{*}, y^{\prime}$-axis perpendicular on $e_{j, k}$ and $x^{\prime}$ axis lying on $e_{j, k}$.

\subsection{Interconnection step}

Previous steps created piercing points that are to be inserted into the coarse mesh to form a higher resolution mesh. Also new edges are inserted by a local triangulation of each coarse triangle. Each coarse triangle is split into 4 subtriangles. The subtriangles are formed from the parent triangles vertices and the piercing points corresponding with each triangle edge to form a conformal triangulation. This yields four possible triangle splits. The choice depends on which of the possible connections minimizes an error metric. We will use the $L_{2}$-error in the remainder of the paper.

Digital images For digital images the algorithm previously described has to be adapted. An earlier paper [15] gives an the algorithm that can be applied to the specific setting of digital images. Next to normal offsets, also vertical offsets $v$ have to be introduced to ensure perfect reconstruction. Also the underlying datastructures which allow for an efficient decomposition are studied. The preliminary results given in [15] indicate that for images of geometrical nature the normal offset decomposition is a promising compression technique.

\section{4. $n$-term approximation}

This paper focuses on a special class of geometrical images. The class of Horizon images $\mathcal{H}$ existing of two constant colored regions separated by one smooth line discontinuity:

$$
\begin{gathered}
\mathcal{H}=\left\{f_{\Omega}(x, y)_{c(x)}\right\} \mid c(x) \in \mathcal{C}^{2} \& \\
f_{\Omega}(x, y)_{c(x)}=\left\{\begin{array}{cc}
H & c(x)>y \\
0 & c(x) \leq y
\end{array}, H \in \mathbf{R} .\right.
\end{gathered}
$$



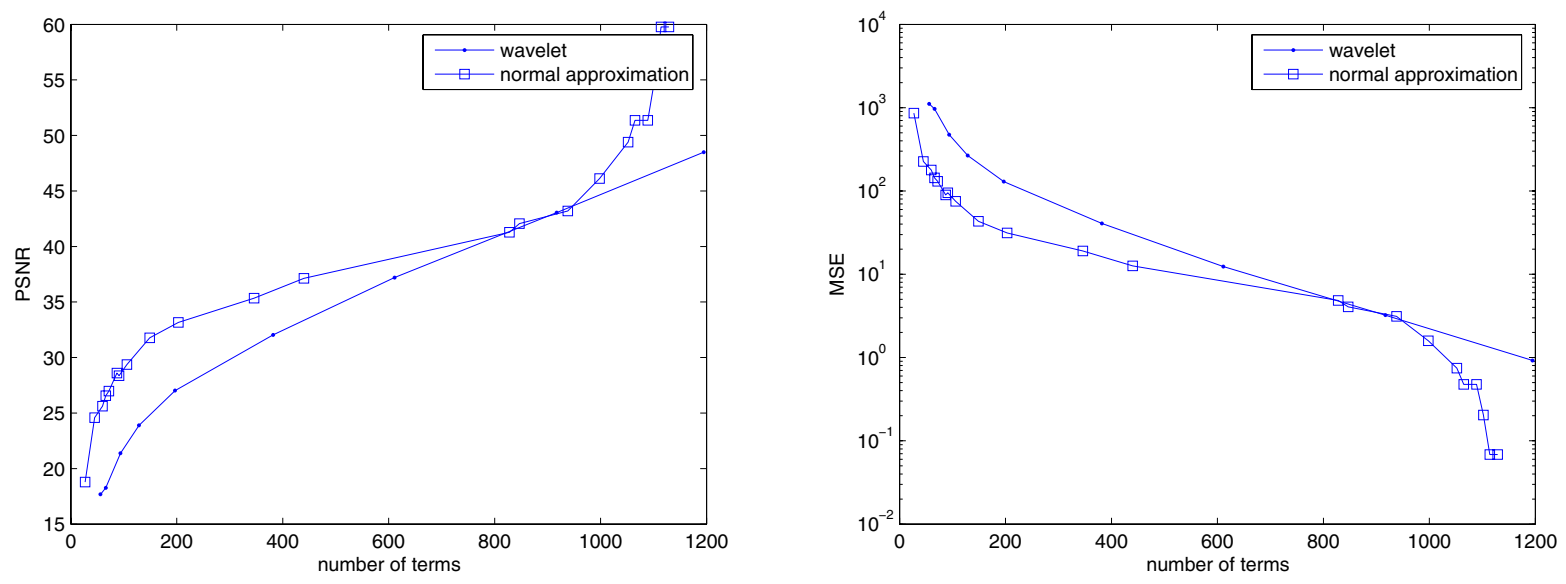

Figure 3. The approximation rate of a wavelet and a normal offset transform on an Horizon type image.

Using a wavelet transform combined with a nonlinear thresholding step, the highest achievable $n$-term approximation rate equals $\sigma_{L_{2}}=O\left(n^{-\frac{1}{2}}\right)$. In [9] it is proven that using their version of a normal offset based method achieves and $n$-term approximation rate equal to $\sigma_{L_{2}}=O\left(n^{-1}\right)$.

\subsection{Experimental comparison with wavelet approximation}

We compare the approximation performance of both a nonlinear wavelet transform (the nonlinearity is introduced by the thresholding step) and the proposed normal offset transform of which both the transform as the $n$-terms selection is nonlinear. For our experiments we used the horizon image shown in Figure 1. A 7-level wavelet decomposition was performed using the daubechies $7 / 9$ wavelet basis. The number of nonzero wavelet coefficients are counted after an hard thresholding step. For the normal offset transform the number of nonzero normal and vertical offsets are counted. The results are given in Figure 3. From a certain amount of terms the rate at which the normal offset method approximates the image outperforms the one of the wavelet method. Figure 4 compares the output images of both nonlinear transforms using the same amount of terms. The wavelet approximated image shows ringing artifacts around the contour.

\section{Compression}

Next subsections derive probability density functions for both vertical and normal offsets. This will allow for an entropy coder to efficiently allocate codewords to the transform coefficients.
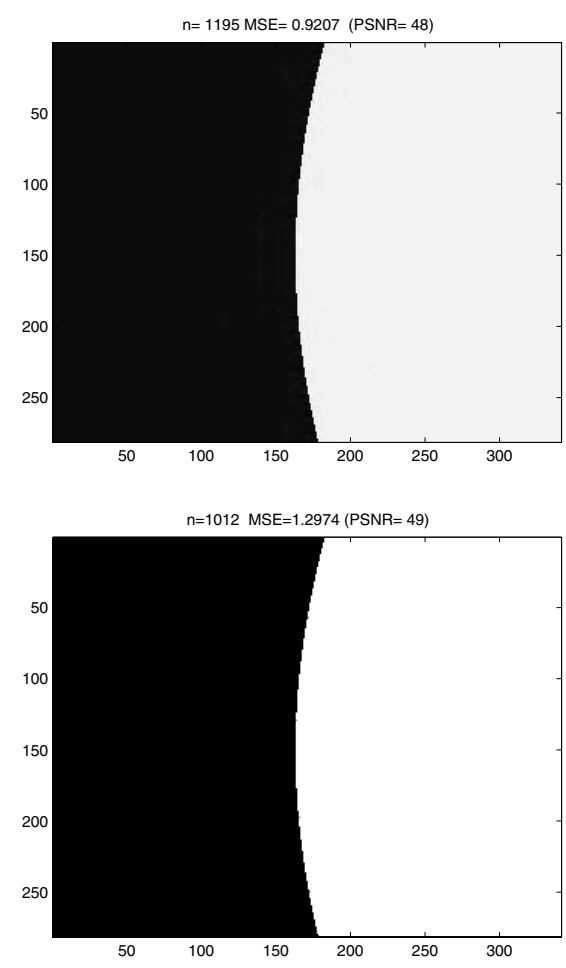

Figure 4. Two examples presenting a reconstructed image of a wavelet transform and a normal offset transform using approximately the same number of terms. 


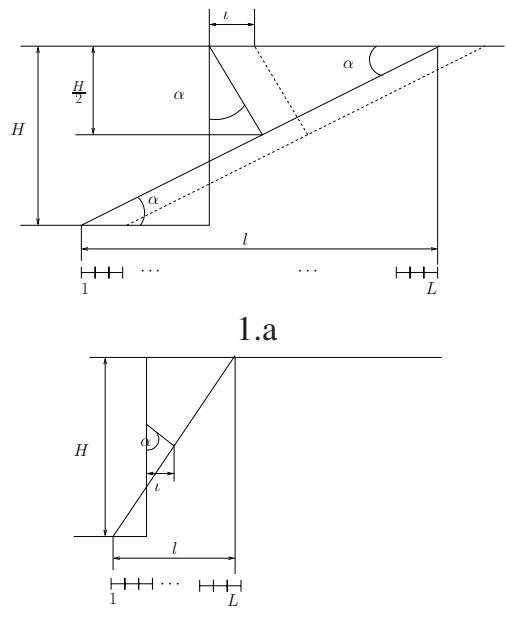

$1 . \mathrm{b}$
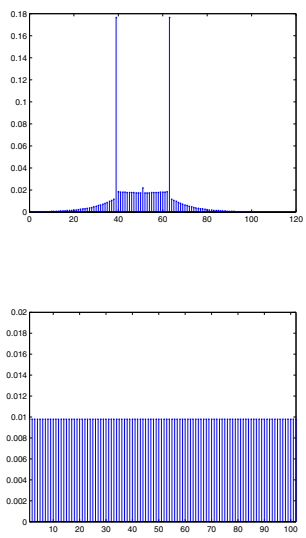

both sides
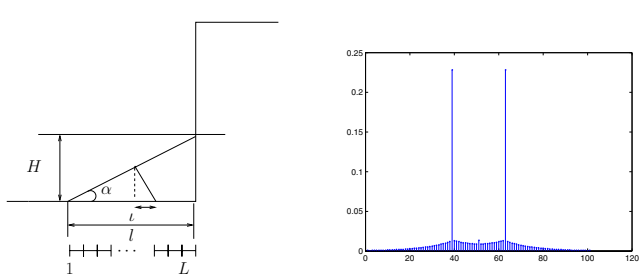

2.a
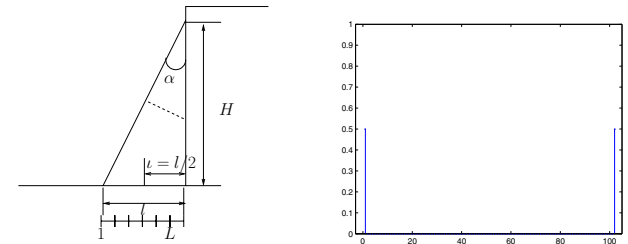

2.b

Figure 5. Four edge/step function situations together with their associated PMF for the normal indices. The two situations on the left of the figure assume a contour running through the vertical plane containing edge $e$. The two situations on the right involve an edge where one of the end points is located on the contour. The $a$-part of each group concerns an edge where $H<l$ where the $b$-part concerns edges where $H \geq l$.

\subsection{Model for the vertical offsets}

The detail information or the high-frequency part of the input signal is gathered into vertical offsets, i.e. the difference between the value of the piercing point and the real function value. According to [13] and confirmed by the experiments shown in Figure 6, the vertical offsets v, can be modeled by a two-parametric zero inflated geometrical distribution $\operatorname{ZID}(p, \lambda)$ (see [1] and references therein) given by:

$$
v \sim f_{V}(v)=p \delta(v)+(1-p) \lambda / 2(1-\lambda)^{v} .
$$

The parameters $p$ and $\lambda$ can be obtained using a mostlikelihood estimate (MLE) method.

\subsection{Model for the normal offsets}

Working with function on the plane we can use the projection of the normal offsets on the $x y$-plane, which makes it easier to derive probability density functions modeling their behavior in the presence of a contour. The projections will further be called normal indices, and be denoted by $i$.

The normal indices can be encoded directly with codewords of $\left\lceil\log _{2} L_{j, k}\right\rceil$ bits, with $L_{j, k}$ the number of pixels in the edge $e_{j, k}$ decaying like $O\left(2^{-j}\right)$. This way of encoding the normal indices presupposes that those indices are distributed uniformly. A uniform distribution, however, implies a maximum entropy. Put differently, when discrete

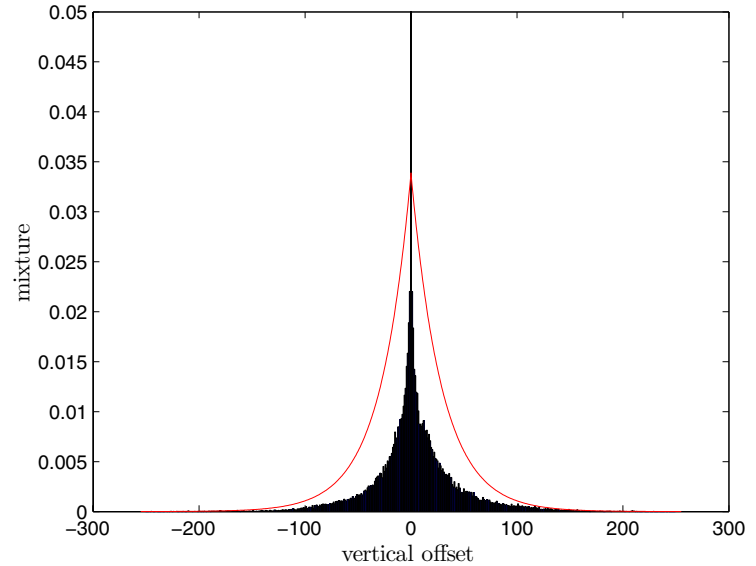

Figure 6. Histogram of the vertical offsets coming from a large test set of geometrical images. The figure suggests a zero inflated double geometrical distribution to model the statistics of the vertical offsets. The parameters produced by the MLE procedure are $p=0.3955$ and $\lambda=0.0339$.

data comes from a uniform distribution the number of expected bits per codeword reaches its maximum. 
We derive an appropriate PMF associated with $i$ for the class of Horizon images which allows an entropy encoder such as an Huffman encoder to lower the expected bitrate. Previous section introduced a model for the vertical offsets $v$. From this model we can deduce a model for the normal indices. The model will depend on wether a triangle edge is crossed by a contour, is flat, or lies with one of its endpoints at a singularity and on the slope of the edge.

It can easily be shown [14] that for the continuous setting, the $P D F$ of $i_{j+1,2 k+1}$ depicted in Figure 5 1.a:

$$
\begin{aligned}
& p\left(i_{j+1,2 k+1}\right)= \\
& \left\{\begin{array}{l}
1 / L \text { iff } i_{j+1,2 k+1} \in\{-\lfloor H \tan \alpha / 2\rfloor, \ldots,\lfloor H \tan \alpha / 2\rfloor\} \\
\frac{1}{2}\left(1-\frac{1}{L}(2\lfloor H \tan \alpha / 2\rfloor+1)\right) \text { iff } i_{j+1,2 k+1}= \pm\lceil H \tan \alpha / \\
0 \text { iff }\left|i_{j+1,2 k+1}\right| \geq\lfloor H \tan \alpha / 2\rfloor
\end{array}\right.
\end{aligned}
$$

For the digital setting we have to introduce vertical offsets and obtain similar PMF models which are discretized versions of $f_{i}(i)=f_{i}\left(i \mid V_{0}=v_{0}, V_{1}=v_{1}\right) * f_{V_{0}}\left(v_{0}\right) * f_{V_{1}}\left(v_{1}\right)$, with $v_{0}$ and $v_{1}$ the vertical offsets at both endpoints of the edge. The different models are depicted in Figure 5.

\section{Conclusions and Results}

This paper presents a nonlinear normal mesh based transform allowing sparse representations of smooth functions containing smooth line singularities. Combined with appropriate distribution models for the normal offset coefficients, the transform can be used in a transform coder. Figure 7 compares our encoder with the JPEG2000 standard on a simple geometrical image. This preliminary result seems promising since the encoding can still be enhanced by including rate-distortion optimized quantizer on the vertical offsets.

\section{Acknowledgments}

The work is supported by the Fund for Scientific Research (FWO) project SMID: Stability of Multiscale Transforms on Irregular Data, grant \#G.0431.05 and the Belgian Programme on Interuniversity Poles of Attraction, initiated by the Belgian Federal Science Policy Office and by the Center Of Excellence on Optimization in Engineering of the K.U.Leuven.

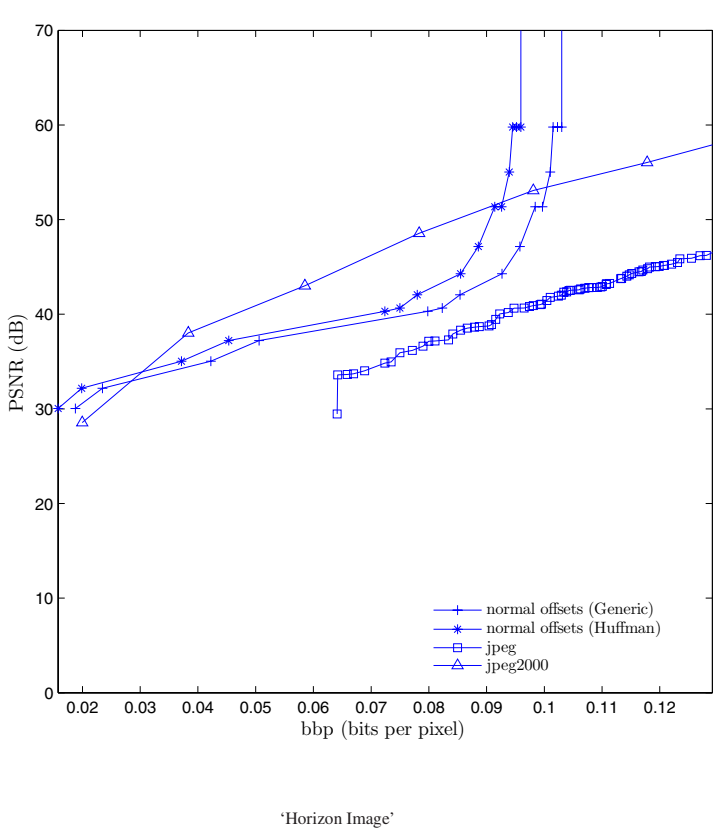

Figure 7. Using the picture of Figure 1 the rate distortion curve of a JPEG encoder and a JPEG2000 encoder together with the rate distortion curve of a normal offset encoder without using offset modeling (Generic) and a normal offset encoder using the proposed models as input for an Huffman entropy encoder (Huffman) is shown. 


\section{References}

[1] D. Agarwal, A. Gelfand, and S. Citron-Pousty. Zero-inflated models with application to spatial count data. Environmental and Ecological Statistics, 9(4):pp. 341-355, December 2002.

[2] P. S. A.Khodakovsky and W. Sweldens. Progressive geometry compression. In K. Akeley, editor, Siggraph 2000, Computer Graphics Proceedings, pages 271-278. ACM Press / ACM SIGGRAPH / Addison Wesley Longman, 2000.

[3] E. J. Candès and D. L. Donoho. Curvelets - a surprisingly effective nonadaptive representation for objects with edges. Technical report, Department of Statistics, Stanford University, 2000.

[4] I. Daubechies, O. Runborg, and W. Sweldens. Normal polyline approximation. Constructive Approximation, 20(3):399-463, May 2004.

[5] S. Dekel and D. Leviatan. Adaptive multivariate approximation using binary space partitions and geometric wavelets. SIAM J. Numer. Anal., 43(2):707-732, 2005.

[6] R. A. DeVore. Nonlinear approximation. Acta Numerica, 7:51-150, 1998.

[7] M. N. Do and M. Vetterli. Contourlets. In G. Welland, editor, Beyond Wavelets, pages 83-107. Academic Press, 2003.

[8] I. Guskov, K. Vidimče, W. Sweldens, and P. Schröder. Normal meshes. In SIGGRAPH 2000 Conference Proceedings, 2000.

[9] M. Jansen, R. Baraniuk, and S. Lavu. Multiscale approximation of piecewise smooth two-dimensional functions using normal triangulated meshes. Appl. Comp. Harm. Anal., 2005.

[10] A. Khodakovsky and I. Guskov. Compression of normal meshes. In Geometric Modeling for Scientific Visualization, pages 189-207. Springer Verlag, 2003.

[11] E. L. Pennec and S. Mallat. Sparse geometric image representation with bandelets. IEEE Trans. on Image Processing, 14(4):423-438, 2005.

[12] H. C. S. Lavu and R. Baraniuk. Geometry compression of normal meshes using rate-distortion algorithms. In SGP '03: Proceedings of the 2003 Eurographics/ACM SIGGRAPH symposium on Geometry processing, pages 52-61, Aire-laVille, Switzerland, Switzerland, 2003. Eurographics Association.

[13] E. Simoncelli. Modeling the joint statistics of images in the wavelet domain. In M. A. Unser, A. Aldroubi, and L. A. F., editors, Wavelet Applications in Signal and Image Processing VII, volume 3813 of SPIE Proceedings, pages 206-214, July 1999.

[14] J. M. Van Aerschot, W. and A. Bultheel. Digital normal meshes in image compression. Technical Report 446, Department of Computer Science, K.U.Leuven, Januari 2006.

[15] W. Van Aerschot, M. Jansen, E. Vanraes, and A. Bultheel. Image compression using normal mesh techniques. In A. Cohen and L. Schumaker, editors, Curves and Surfaces, Avignon 2006, Brentwood, 2006. Nashboro Press. Accepted. 